\section{Crescimento de pacientes com hiperplasia congênita das supra-renais, forma perdedora de sal, nos dois primeiros anos de vida}

\section{Growth in patients with the salt-wasting form of congenital adrenal hyperplasia during the first two years of life}

Carolina T. Mendes-dos-Santos 1

Sofia H. V. de Lemos-Marini 2

Maria Tereza M. Baptista 3

Gil Guerra-Junior 4

Maricilda P. De-Mello 5

André M. Morcillo 6
Abstract

Objectives: to assess the growth and nutritional recovery of patients with the classical salt-wasting form of congenital adrenal hyperplasia in the first two years of life.

Methods: z scores for weight and height were calculated for 21 patients at birth, on the occasion of the first medical consultation and at one and two years of age. The concentrations of 17-hydroxyprogesterone, androstenedione and the doses of hydrocortisone prescribed at the first medical concentrations up to the age of two years were determined (at one and two years of age respectively).

Results: the mean age for the first medical consultation was 36.7 days. The $z$ score for weight at birth was $-0.23 \pm 1.4$; on the occasion of the first consultation $-2.31 \pm 1.3$; at the age of one year $-1.43 \pm 1.6$ and at the age of two years $-0.77 \pm 1.3$. The $z$ score for

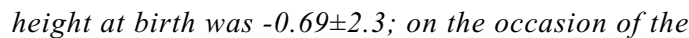
first consultation $-1.87 \pm 1.7$; at one year of age $1.68 \pm 1.1$ and at two years $-1.07 \pm 1.0$. The difference between the scores at two years of age and on the occasion of the first medical consultation was $1.54 \pm 1.7$ for weight and $0.80 \pm 1.6$ for height. The mean dosage of hydrocortisone prescribed was 21.3 and $19.9 \mathrm{mg} / \mathrm{m} 2 /$ day for periods 1 and 2 and the concentrations ( $\mathrm{ng} / \mathrm{dL}$ ) of 17-hydroxyprogesterone and androstenedione were 9.1 and 0.14 for period 1 and 4.4 and 0.27 for period 2 .

Conclusions: nutritional recovery was observed to occur on treatment and, at two years of age, weight and height are normal, although below the average for the population at large.

Key words Growth, Adrenal hyperplasia, congenital, Adrenal glands

\section{Resumo}

Objetivos: avaliar crescimento e recuperação nutricional de pacientes com hiperplasia congênita supra-renal, forma clássica perdedora de sal, nos dois primeiros anos de vida.

Métodos: analisamos escores $z$ de peso e comprimento de 21 pacientes ao nascimento, primeira consulta, com um e dois anos de idade. Determinamos concentrações de 17-hidroxiprogesterona, androstenediona e doses de hidrocortisona prescritas da primeira consulta até um e dois anos de idade (periodos 1 e 2, respectivamente).

Resultados: a média de idade na primeira consulta foi 36,7 dias. Escore $z$ do peso ao nascimento foi $-0,23 \pm 1,4$; na primeira consulta $-2,31 \pm 1,3$; com um ano $-1,43 \pm 1,6$ e dois anos $-0,77 \pm 1,3$. Escore $z$ do comprimento ao nascimento foi $-0,69 \pm 2,3$; na primeira consulta $-1,87 \pm 1,7$; com um ano $-1,68 \pm 1,1 e$ dois anos $-1,07 \pm 1,0$. A diferença entre os escores aos dois anos e na primeira consulta foi 1,54 $\pm 1,7$ para o

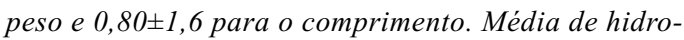
cortisona prescrita foi 21,3 e 19,9 mg/m2/dia nos períodos 1 e 2 e concentrações (ng/dL) de 17-hidroxiprogesterona e androstenediona foram 9,1 e 0,14 no periodo 1 e 4,4 e 0,27 no 2, respectivamente.

Conclusões: foram observados recuperação nutricional com o tratamento $e$, aos dois anos, peso $e$ comprimento normais, embora inferiores aos da população.

Palavras-chave Crescimento, Hiperplasia suprarenal congênita, Glândulas supra-renais 


\section{Introdução}

A hiperplasia congênita das supra-renais (HCSR) por deficiência de 21-hidroxilase $(21 \mathrm{OH})$ é doença autossômica recessiva, na qual ocorre diminuição da síntese de glicocorticóides e aumento da produção de andrógenos. A incidência da sua forma clássica varia entre 1:12.000 e 1:15.000 e, em aproximadamente $75 \%$ dos casos, a variante clínica é a perdedora de sal. 1

Embora pacientes com HCSR-21OH possam apresentar desnutrição e retardo de crescimento devido à perda de sal ou às infecções intercorrentes, a maturação esquelética pode prosseguir seu desenvolvimento normal ou até de forma mais avançada devido ao aumento de andrógenos e, consequentemente, a estatura final pode ser comprometida. ${ }^{2}$

Além disso, com o avanço da idade óssea, o paciente pode desenvolver puberdade precoce central secundária à puberdade precoce periférica devido à HCSR, e ter seu crescimento precocemente interrompido. 3

O tratamento consiste na reposição de doses fisiológicas de glicocorticóide e, quando necessário, de mineralocorticóide.4,5 Procura-se usar sempre a menor dose de glicocorticóide que possa garantir controle do hiperandrogenismo.6,7 A maior dificuldade está em alcançar a dose ótima, capaz de promover crescimento o mais próximo do normal.811 Doses insuficientes de glicocorticóide provocam aumento na produção de esteróides sexuais, avanço da idade óssea e fechamento precoce das epífises ósseas, ao passo que o excesso de glicocorticóides pode suprimir o crescimento. 12

Existem controvérsias referentes ao efeito do início precoce da terapia, enquanto alguns autores consideram que o mesmo pode minimizar os efeitos da doença sobre o crescimento, 2,8,13-17 outros não encontraram tal correlação. 18

Alguns trabalhos avaliaram o crescimento de pacientes com HCSR-21OH desde o início do tratamento até a altura adulta, mas são poucos os dados disponíveis dos primeiros anos de vida. A maioria mostra que a altura final encontra-se abaixo da altura da população geral e dos alvos parentais. ${ }^{14,16,18}$

O objetivo deste estudo foi avaliar o crescimento e a recuperação nutricional de pacientes com HCSR$21 \mathrm{OH}$, forma clássica perdedora de sal, em seus dois primeiros anos de vida.

\section{Métodos}

Estudo retrospectivo em que foram incluídas as 21 crianças com HCSR-21OH, forma clássica perde- dora de sal; com idade máxima de início de seguimento de quatro meses; acompanhadas no Serviço de Endocrinologia Pediátrica do Hospital das Clínicas da Universidade Estadual de Campinas (HC/UNICAMP) São Paulo, Brasil, até completarem dois anos de idade, no período de 1993 a 2003.

O tratamento foi supervisionado por um único médico durante todo o período de estudo, com média de dez avaliações no primeiro ano e cinco no segundo. Todos os pacientes foram tratados somente com acetato de hidrocortisona na dose de 17 a 23 $\mathrm{mg} / \mathrm{m}^{2} \mathrm{SC} /$ dia (três tomadas diárias iguais) e fludrocortisona na dose de 0,05 a $0,1 \mathrm{mg} / \mathrm{dia}$ (uma ou duas tomadas diárias). Adicionalmente, durante $\mathrm{o}$ primeiro ano, receberam suplementação de sal através da oferta de solução salina a $1 \%$ entre as refeições, sem restrições de quantidade.

Foram critérios de exclusão: tratamento com glicocorticóide de meia vida média ou longa e início do seguimento no referido serviço após o quarto mês de vida.

O diagnóstico da HCSR foi realizado para todos os sujeitos a partir da determinação sérica de 17hidroxiprogesterona por radioimunoensaio, fase sólida, utilizando-se o kit comercial Diagnostic Systems Laboratories (DSL), e confirmado por estudo de biologia molecular. Para definição da forma perdedora de sal, foram avaliados a evolução do peso prévia ao início do tratamento, os valores plasmáticos de sódio e potássio ao diagnóstico e a presença de desidratação.

As informações referentes a sexo, etnia, data de nascimento, além de peso e comprimento (ao nascer, na primeira consulta, com um e dois anos de idade), foram obtidas através dos prontuários.

Foram considerados dois períodos de investigação: "período 1", que correspondeu ao intervalo de tempo desde a primeira consulta na UNICAMP até a data de consulta mais próxima ao primeiro aniversário do paciente e "período 2", referente ao intervalo de tempo desde o início do tratamento no Serviço até a data de consulta mais próxima ao segundo aniversário. Determinou-se a dose média de hidrocortisona prescrita no período 1 e no período 2 , e na avaliação das concentrações da 17-hidroxiprogesterona e da androstenediona nos dois períodos, optou-se pelo uso da mediana dos seus valores, para contornar o problema dos limites inferior $(<0,01$ $\mathrm{ng} / \mathrm{mL}$ ) e superior ( $>10 \mathrm{ou}>20 \mathrm{ng} / \mathrm{mL}$ ) do método, cujos valores reais eram desconhecidos.

Para determinar a idade óssea aos dois anos utilizou-se a primeira avaliação realizada com idade igual ou superior a 24 meses, ajustando através de regra de três simples a idade óssea para a idade 
cronológica de dois anos.

O peso e o comprimento foram transformados em escores z, usando-se os dados de referência do National Center for Health Statistics (CDC 2000)19 Os dados são apresentados em tabelas e figuras contendo os valores da média, desvio padrão, percentis 25,50 e 75 . Na análise dos dados empregou-se o teste de Friedman, com nível de significância estatística de 5\%.

Este trabalho foi aprovado pelo Comitê de Ética em Pesquisa da Faculdade de Ciências Médicas da UNICAMP (processo 120/2003) e um dos pais ou o responsável pelo paciente assinou o termo de consentimento pós-informado permitindo o estudo.

\section{Resultados}

Entre os 21 pacientes, com média de idade na primeira consulta de 36,7 dias (3 a 123), 13 (61,9\%) são do sexo feminino; a idade de início de tratamento foi menor nas meninas (20,8 dias) que nos meninos (50,1 dias). A dose média de hidrocortisona $\left(\mathrm{mg} / \mathrm{m}^{2} / \mathrm{dia}\right)$ foi $21,3(18,2$ a 23,4$)$ no período $1 \mathrm{e}$ 19,9 (17 a 22,6) no período 2.

A concentração da 17-hidroxiprogesterona (ng/dL) no período 1 foi $9,1(0,2$ a 41,6), enquanto no período 2 foi $4,4(0,12$ a 25$)$; a de androstenediona $(\mathrm{ng} / \mathrm{dL})$ foi $0,14(0,01$ a 0,5$)$ e $0,27(0,01$ a $3,07)$ respectivamente, nos períodos 1 e 2 .

A média da idade óssea aos dois anos foi 1,6 anos $(0,6$ a 2,5$)$.

A média dos escores $\mathrm{z}$ do peso ao nascimento foi $-0,23$; na primeira consulta $-2,31$; com um ano de idade $-1,43$ e aos dois anos $-0,77(p<0,001)$, conforme apresentado na Tabela 1 e Figura 1 . A média da diferença entre os escores $\mathrm{z}$ de peso na idade de dois anos e na primeira consulta foi 1,54. A média dos escores $\mathrm{z}$ do comprimento ao nascimento foi $-0,69$; na primeira consulta $-1,87$; com a idade de um ano $-1,68$ e aos dois anos de idade $-1,07$ $(p=0,029)$, conforme apresentado na Tabela $2 \mathrm{e}$ Figura 2. A média da diferença entre os escores $\mathrm{z}$ de comprimento na idade de dois anos e na primeira consulta foi 0,80 .

\section{Tabela 1}

Valores médios $(\bar{X} \pm D P$ ) e percentis 25,50 e 75 dos escores $z$ do peso para idade.

\begin{tabular}{|c|c|c|c|c|}
\hline & $\overline{\mathbf{X}} \pm \mathrm{DP}$ & P25 & P50 & P75 \\
\hline \multicolumn{5}{|c|}{ Masculino $(n=8)$} \\
\hline Nascimento & $0,15 \pm 1,3$ & $-0,98$ & $-0,22$ & 1,32 \\
\hline $1^{\text {a }}$ consulta & $-2,65 \pm 1,7$ & $-3,28$ & $-2,34$ & $-1,38$ \\
\hline 1 ano & $-1,89 \pm 1,8$ & $-2,13$ & $-1,51$ & $-0,76$ \\
\hline 2 anos & $-1,16 \pm 1,6$ & $-1,66$ & $-0,87$ & 0,02 \\
\hline \multicolumn{5}{|c|}{ Feminino $(n=13)$} \\
\hline Nascimento & $-0,46 \pm 1,5$ & $-1,66$ & $-0,29$ & 0,89 \\
\hline $1^{\text {a }}$ consulta & $-2,10 \pm 1,0$ & $-2,66$ & $-2,20$ & $-1,46$ \\
\hline 1 ano & $-1,15 \pm 1,6$ & $-2,22$ & $-0,99$ & $-0,11$ \\
\hline 2 anos & $-0,54 \pm 1,2$ & $-1,59$ & $-0,49$ & $-0,07$ \\
\hline \multicolumn{5}{|c|}{ Todos os casos $(n=21)$ * } \\
\hline Nascimento & $-0,23 \pm 1,4$ & $-1,10$ & $-0,29$ & 1,04 \\
\hline $1^{\text {a }}$ consulta & $-2,31 \pm 1,3$ & $-2,69$ & $-2,20$ & $-1,46$ \\
\hline 1 ano & $-1,43 \pm 1,6$ & $-2,21$ & $-1,27$ & $-0,36$ \\
\hline 2 anos & $-0,77 \pm 1,4$ & $-1,59$ & $-0,77$ & 0,01 \\
\hline
\end{tabular}

$\mathrm{P} 25=$ percentil $25 ; \mathrm{P} 50=$ percentil $50 ; \mathrm{P} 75=$ percentil $75 ;{ }^{*} p<0,001-$ Teste de Friedman. 
Figura 1

Intervalos de confiança de $95 \%$ da média dos escores $z$ do peso.

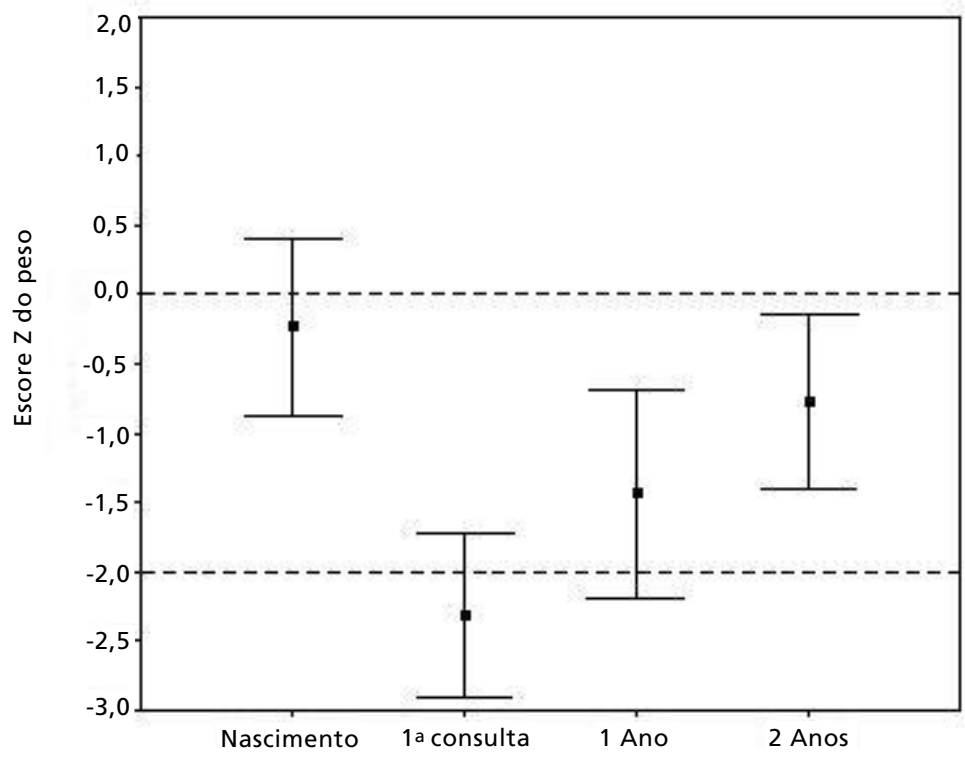

Tabela 2

Valores médios ( $\bar{X} \pm$ DP ) e percentis 25,50 e 75 dos escores $z$ do comprimento para idade.

\begin{tabular}{|c|c|c|c|c|}
\hline & $\bar{X} \pm D P$ & P25 & P50 & P75 \\
\hline \multicolumn{5}{|c|}{ Masculino $(n=8)$} \\
\hline Nascimento & $-0,90 \pm 0,8$ & $-1,74$ & $-0,90$ & $-0,06$ \\
\hline $1^{\text {a }}$ consulta & $-2,24 \pm 2,0$ & $-3,13$ & $-1,31$ & $-1,09$ \\
\hline 1 ano & $-1,67 \pm 1,3$ & $-2,94$ & $-1,87$ & $-0,23$ \\
\hline 2 anos & $-1,34 \pm 1,3$ & $-2,04$ & $-1,60$ & $-0,17$ \\
\hline \multicolumn{5}{|c|}{ Feminino $(n=13)$} \\
\hline Nascimento & $-0,58 \pm 2,8$ & $-2,99$ & $-0,19$ & 2,15 \\
\hline $1^{\text {a }}$ consulta & $-1,64 \pm 1,5$ & $-2,17$ & $-1,64$ & $-0,31$ \\
\hline 1 ano & $-1,68 \pm 1,1$ & $-2,55$ & $-1,48$ & $-0,72$ \\
\hline 2 anos & $-0,91 \pm 0,8$ & $-1,46$ & $-0,76$ & $-0,30$ \\
\hline \multicolumn{5}{|c|}{ Todos os casos $(n=21)$ * } \\
\hline Nascimento & $-0,69 \pm 2,3$ & $-2,06$ & $-0,62$ & 0,75 \\
\hline $1^{\text {a }}$ consulta & $-1,87 \pm 1,7$ & $-2,17$ & $-1,39$ & $-0,79$ \\
\hline 1 ano & $-1,68 \pm 1,2$ & $-2,69$ & $-1,66$ & $-0,72$ \\
\hline 2 anos & $-1,07 \pm 1,0$ & $-1,82$ & $-0,92$ & $-0,30$ \\
\hline
\end{tabular}

$\mathrm{P} 25=$ percentil $25 ; \mathrm{P} 50=$ percentil $50 ; \mathrm{P} 75=$ percentil $75 ;{ }^{*} p<0,029-$ Teste de Friedman. 
Intervalos de confiança de $95 \%$ da média dos escores z do comprimento.

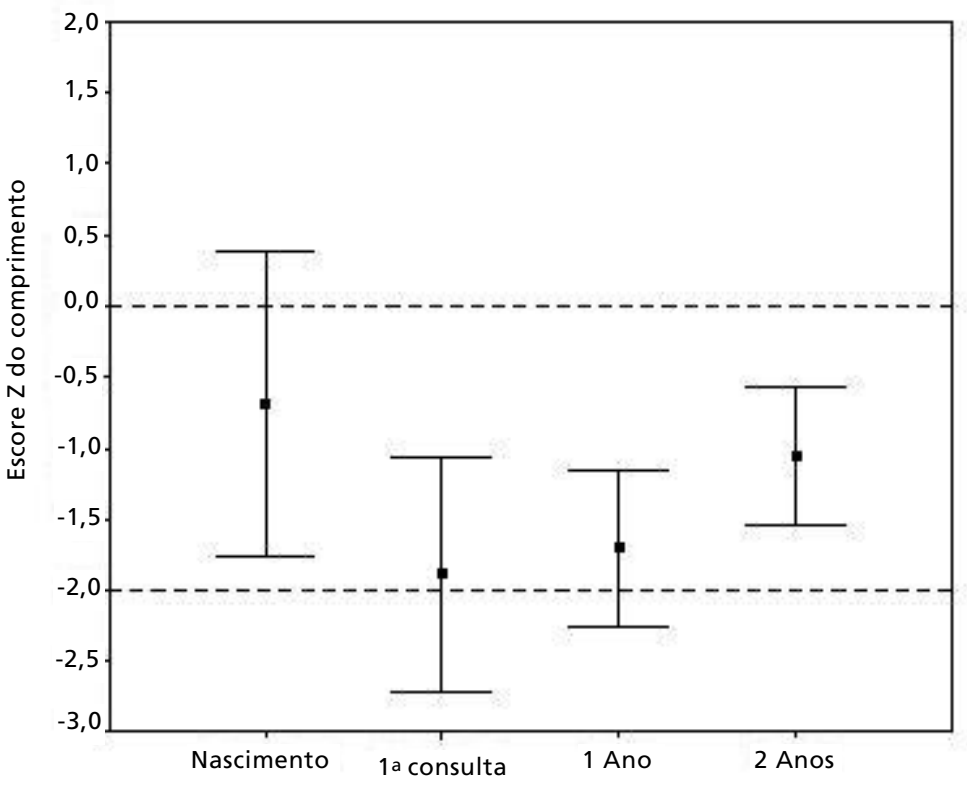

Observa-se que os pacientes apresentam piora dos dados antropométricos do nascimento até a primeira consulta, com recuperação progressiva após o início do tratamento. Não houve correlação entre o peso e o comprimento com um e dois anos de idade e as doses prescritas de hidrocortisona ou as concentrações séricas de 17-hidroxiprogesterona e androstenediona no período 1 ou no 2 . A diferença entre peso e comprimento aos dois anos e na primeira consulta também não mostrou correlação com as doses de hidrocortisona ou com as concentrações séricas de 17-hidroxiprogesterona e androstenediona nos períodos estudados.

\section{Discussão}

Embora diversos autores apontem para diminuição de estatura final dos pacientes com HCSR, 7,8,13 este estudo mostra recuperação do peso e do comprimento já nos primeiros dois anos de vida, sem comprometimento da maturação esquelética.

A maioria dos estudos sobre crescimento em HCSR avalia a estatura final dos pacientes, entretanto, poucos autores analisaram evolutivamente o período que vai do início do tratamento até o momento em que os pacientes atingem a altura final. Além disso, diversos trabalhos avaliam conjuntamente pacientes com as formas clássicas (perdedora de sal e virilizante simples) ${ }^{16,18}$ e mesmo com a forma não clássica, 15 embora o comportamento de cada uma seja bastante diverso.

Um dos trabalhos que avaliou o crescimento de crianças com HCSR-21 foi o de Manoli et al., 14 desenvolvido em Atenas: estudaram 48 pacientes de um único serviço, contudo, somente dez dos perdedores de sal (dois meninos e oito meninas) apresentavam dados dos dois primeiros anos de vida. Apesar da média do comprimento aos dois anos para os meninos $(\mathrm{M})$ e meninas $(\mathrm{F})$ ter sido maior $(\mathrm{M}=$ $-0,45 \pm 0,2 / \mathrm{F}=-0,33 \pm 0,9)$ que a observada no nosso grupo $(\mathrm{M}=-1,34 \pm 1,3 / \mathrm{F}=-0,91 \pm 0,8)$, os autores não forneceram dados da antropometria ao nascimento e na ocasião do diagnóstico, o que possibilitaria a comparação da evolução com o tratamento. Os autores também referiram que aos dois anos de idade os meninos tinham um ano de avanço da idade óssea em relação à idade cronológica, o que poderia ser decorrente de tratamento com doses insuficientes de glicocorticóides.

No estudo de Van der Kamp et al., 16 realizado na Holanda, os pacientes (12 meninas e 20 meninos) são provenientes de seis serviços. $\mathrm{Na}$ ocasião do diagnóstico apresentavam comprimento menos afetado $(\mathrm{M}=-0,87 \pm 0,93 / \mathrm{F}=-0,27 \pm 0,97)$ que os pacientes do presente estudo $(\mathrm{M}=-2,24 \pm 2,0 / \mathrm{F}=$ $-1,64 \pm 1,5)$. No entanto, apresentaram piora do 
escore de comprimento nos três primeiros meses, atribuída às altas doses de glicocorticóides utilizadas (cerca de $40 \mathrm{mg} / \mathrm{m}^{2} \mathrm{SC} / \mathrm{dia}$ ). A comparação entre os dois estudos não é simples, pelo fato de que nem todas as crianças do grupo holandês receberam suplementação salina. $\mathrm{O}$ escore $\mathrm{z}$ de comprimento dos nossos pacientes na idade de dois anos $(-1,07)$ é melhor que o dos pacientes do referido trabalho sem suplementação salina $(-1,56)$, e semelhante aos daqueles que a receberam $(-1,0)$.

Hargitai et al. 18 estudaram o crescimento de crianças portadoras de HCSR de cinco países europeus, mas referem ter poucos dados dos primeiros três anos de vida. Seus pacientes apresentaram padrão de crescimento piores que os do estudo atual, sendo que os meninos perdedores de sal encontravam-se no percentil 5 da curva de referência com 18 meses, enquanto as meninas tinham altura no mesmo percentil aos três anos de idade.

Estudo realizado anteriormente em nosso serviço 20 também mostrou melhora dos escores $\mathrm{z}$ de peso e estatura no decorrer do tratamento, mas o grupo de pacientes também era heterogêneo, constituído por 45 pacientes ( 31 meninas e 14 meninos) com a forma clássica da HCSR e idades entre 2,8 e 26,6 anos, sendo 30 com a variante perdedora de sal e 15 com a virilizante simples, com idade na consulta inicial entre 0 e 13,9 anos. Além disso,

\section{Referências}

1. American Academy of Pediatrics. Technical report: Congenital adrenal hyperplasia. Pediatrics. 2000; 106: 1511-8.

2. Nguyen AT, Brown JJ, Warne GL. Growth in congenital adrenal hyperplasia. Indian J Pediatr. 2006; 73: 89-93.

3. New MI. An update of congenital adrenal hyperplasia. Ann N Y Acad Sci. 2004; 1038: 14-43.

4. Pang S. Congenital adrenal hyperplasia. Endocrinol Metab Clin North Am. 1997; 26: 853-91.

5. Forest MG. Recent advances in the diagnosis and management of congenital adrenal hyperplasia due to 21-hydroxylase deficiency. Hum Reprod Update. 2004; 10: 469-85.

6. Miller WL. Genetics, diagnosis, and management of 21hydroxilase deficiency. J Clin Endocrinol Metab. 1994; 78: 241-6.

7. Girgis R, Winter JSD. The effects of glucocorticoid replacement therapy on growth, bone mineral density, and bone turnover markers in children with congenital adrenal hyperplasia. J Clin Endocrinol Metab. 1997; 82:3 926-9.

8. Eugster EA, Dimeglio LA, Wright JC, Freidenberg GR, Seshadri R, Pescovitz OH. Height outcome in congenital adrenal hyperplasia caused by 21 hydroxylase deficiency: a meta-analysis. J Pediatr. 2001; 138: 26-32. foram incluídos pacientes tratados com glicocorticóides de meia vida média e longa, com maior efeito inibidor no crescimento.

O grupo que mais se assemelha ao do presente estudo é o de Gussinye et al.,21 realizado em Barcelona, composto por 24 pacientes perdedores de sal (19 meninas e 5 meninos), com diagnóstico até a idade de seis meses. Não há informações quanto aos dados ao nascimento e os de comprimento na ocasião do diagnóstico são melhores que os encontrados em nosso estudo. Eles apresentavam escore $\mathrm{z}$ $0,38 \pm 0,82$, enquanto nossos pacientes tinham $-0,23 \pm 1,4$. Na avaliação do comprimento com um ano, nossos pacientes apresentam-se melhores (escore $\mathrm{z}=-1,68$ ) que os de Gussinye et al. ${ }^{21}$ (escore $\mathrm{z}=-2,21$ ), provavelmente porque, no primeiro e segundo anos de vida, as doses de glicocorticóides utilizadas pelas crianças espanholas (34,53 e 22,83 $\mathrm{mg} / \mathrm{m}^{2} \mathrm{SC} / \mathrm{dia}$ ) foram maiores que as dos nossos pacientes $\left(21,3\right.$ e $\left.18,3 \mathrm{mg} / \mathrm{m}^{2} \mathrm{SC} / \mathrm{dia}\right)$.

O grupo de pacientes estudado mostrou recuperação nutricional progressiva após o início do tratamento e, aos dois anos de idade, o peso e comprimento apresentaram-se dentro dos parâmetros normais, embora abaixo da média da população geral.

9. Cameron FJ, Kaymakci B, Byrt EA, Ebeling PR, Warne GL, WalkJD. Bone mineral density and body composition in congenital adrenal hyperplasia. J Clin Endocrinol Metab. 1995; 80: 2238-43.

10. Cornean RE, Hindmarsh PC, Brook CG. Obesity in 21hydroxylase deficient patients. Arch Dis Child. 1998; 78: 261-3.

11. Migeon CJ, Donohoue PA. Congenital adrenal hyperplasia caused by 21 -hydroxylase deficiency. Its molecular basis and its remaining therapeutic problems. Endocrinol Metab Clin North Am. 1991; 20: 277-96.

12. Merke DP, Bornstein SR. Congenital adrenal hyperplasia. Lancet. 2005; 365: 2125-36.

13. Schwartz RP. Back to basics: early diagnosis and compliance improve final height outcome in congenital adrenal hyperplasia. J Pediatr. 2001; 138: 3-5.

14. Manoli I, Kanaka-Gantenbein CH, Voutetakis A, ManiatiChristidi M, Dacou-Voutetakis A. Early growth, pubertal development, body mass index and final height of patients with congenital adrenal hyperplasia: factors influencing the outcome. Clin Endocrinol (Oxf). 2002; 57: 669-76.

15. Savage MO, Scommegna S, Carroll PV, Ho JT, Monson JP, Besser GM, Grossman AB. Growth in disorders of adrenal hyperfunction. Horm Res. 2002; 58 (suppl 1): 39-43. 
16. Van der Kamp HJ, Otten BJ, Buitenweg N, De Munick Keiser-Schrama SMPF, Oostdijk W, Jansen M, Delemarrede-Waal HA, Vulsma T, Wit JM. Longitudinal analysis of growth and puberty in 21-hydroxylase deficiency patients. Arch Dis Child. 2002; 87: 139-44.

17. Lemos-Marini SHV, Guerra-Júnior G, Morcillo AM, Baptista MTM, Silva LO, Maciel-Guerra AT. Hiperplasia congênita das supra-renais por deficiência da 21-hidroxilase: altura final de 27 pacientes com a forma clássica. Arq Bras Endocrinol Metab. 2005; 49: 902-7.

18. Hargitai G, Sólyom J, Battelino T, Lebl J, Pribilincová Z, Hauspie R, Kovács J, Waldhauser F, Frisch $\mathrm{H}$ and the MEWPE-CAH study group. Growth patterns and final height in congenital adrenal hyperplasia due to classical 21 hydroxylase deficiency. Results of a multicenter study. Horm Res. 2001; 55: 161-71.
19. National Center for Health Statistics. 2000 CDC growth charts: United States. [cited on 2007 mar 8] Available from: http://www.cdc.gov/growthcharts/

20. Ramos CCA, Bento LR, Gonçalves EM, De-Mello MP, Baptista MTM, Lemos-Marini SHV, Guerra-Junior G. Avaliação do crescimento, do controle laboratorial e da corticoterapia em um grupo de pacientes com a forma clássica da deficiência da 21-hidroxilase. Rev Paul Pediatr. 2007; 25: 317-23.

21. Gussinye M, Potau N, Vicens-Calvet E, Albisu MA, Yeste D, Ibanez L, Audí L, Carrascosa A. Adult height, pattern of growth and pubertal development in patients with congenital adrenal hyperplasia, salt losing form. Med Clin (Barc). 1997; 108: 87-90.

Recebido em 15 de outubro de 2008

Versão final apresentada em 5 de agosto de 2009

Aprovado em 28 de setembro de 2009 